\title{
Avaliação das propriedades de painéis aglomerados produzidos com resíduos de serrarias de nove espécies de madeiras tropicais da Amazônia
}

\author{
Setsuo IWAKIRI ${ }^{1}$, Basílio Frasco VIANEZ², Cristiane WEBER ${ }^{3}$, Rosilani TRIANOSKI ${ }^{3}$, Vanessa Coelho \\ ALMEIDA $^{3}$
}

\begin{abstract}
RESUMO
Esta pesquisa teve como objetivo avaliar a qualidade de painéis aglomerados produzidos com resíduos de processamento em serraria de nove espécies de madeiras tropicais da Amazônia. As espécies estudadas foram: Scleronema micranthum Ducke (Cardeiro), Ecclinusa guianensis Eyma (Caucho), Scleronema sp. (Castanha-de-paca), Copaifera multijuga Hayne (Copaíba), Ocotea sp. (Louro), Ocotea guianensis Aubl (Louro-espinho), Caryocar villosum Pers. (Piquiarana), Couratari oblongifolia Ducke $\&$ R. Knuth (Tauari) e Virola surinamensis Rol. Warb (Virola). Foram produzidos painéis experimentais com densidade nominal de $0,75 \mathrm{~g} . \mathrm{cm}^{-3}$, utilizando a resina uréia-formaldeído na proporção de $8 \%$ de sólidos - base peso seco das partículas. Os painéis foram prensados com pressão específica de $4,0 \mathrm{MPa}$, temperatura de $160^{\circ} \mathrm{C}$ e tempo de prensagem de oito minutos. As avaliações dos resultados de ensaios obtidos nesta pesquisa indicam a viabilidade técnica de utilização das nove espécies provenientes de florestas tropicais da Amazônia na produção de painéis de madeira aglomerada, com destaque para Ecclinusa guianensis Eyma (Caucho) que, de uma forma geral, apresentou melhores resultados de propriedades físico-mecânicas.
\end{abstract}

PALAVRAS-CHAVE: Painéis aglomerados, madeiras tropicais, uréia-formaldeído.

\section{Evaluation of the properties of particleboard made from sawmill waste of nine tropical wood species of Amazon}

\begin{abstract}
This research was developed to evaluate the quality of particleboards manufactured from sawmill waste of nine tropical wood species of Amazônia. The following species were studied: Scleronema micranthum Ducke (Cardeiro), Ecclinusa guianensis Eyma (Caucho), Scleronema sp. (Castanha-de-paca), Copaifera multijuga Hayne (Copaíba), Ocotea sp. (Louro), Ocotea guianensis Aubl (Louro-espinho), Caryocar villosum Pers. (Piquiarana), Couratari oblongifolia (Tauari) e Virola surinamensis Rol. Warb (Virola). The experimental boards were manufactured with the nominal density of $0.75 \mathrm{~g} . \mathrm{cm}^{-3}$, using the urea-formaldehyde resin in the proportion of $8 \%$ of solid content based on oven dried wood particles. The boards were pressed at the specific pressure of $40 \mathrm{kgf} / \mathrm{cm}^{2}$, temperature of $160{ }^{\circ} \mathrm{C}$ and press time of eight minutes. The evaluations of test results obtained in this study indicate the potential use of the nine species from Amazon rainforest in the production of particleboard, highlighting Ecclinusa guianensis (Caucho) that, in general, showed better results of physical and mechanical properties.
\end{abstract}

KEYWORDS: Particleboard, tropical woods, urea-formaldehyde.

1 Universidade Federal do Paraná - DETF. E-mail: setsuo@ufpr.br

2 Instituto Nacional de Pesquisas da Amazônia - CPPF. E-mail: basilio@inpa.gov.br

3 Universidade Federal do Paraná - PPGEF. E-mail: criswef@gmail.com, rosillani@gmail.com, vanessaufrrj@yahoo.com.br. 


\section{INTRODUÇÃO}

As madeiras de pinus e eucalipto constituem a base de matéria-prima para produçáo de painéis aglomerados e MDF no Brasil. Nas últimas duas décadas, as indústrias do setor de painéis reconstituídos de madeira tem empreendido grandes investimentos na implantação de novas unidades produtivas, além do aumento em novas áreas de plantios florestais para assegurar o suprimento de madeira. De acordo com o estudo setorial do BNDES (2008), a produção brasileira de painéis aglomerados cresceu de 0,866 milhão de $\mathrm{m}^{3}$. ano ${ }^{-1}$ em 1995 para 2,098 milhóes de $\mathrm{m}^{3}$.ano ${ }^{-1}$ em 2005 . O crescimento médio neste período foi de $9,3 \%$ ao ano, um percentual bem superior ao 4,5\% do nível mundial. Este crescimento deve-se à demanda crescente do setor moveleiro que utiliza amplamente este produto na fabricação de móveis.

As indústrias de painéis aglomerados no Brasil estão localizadas nas regióes sul e sudeste, tendo como foco o atendimento aos pólos moveleiros instalados principalmente nos Estados de São Paulo, Minas Gerais, Paraná, Santa Catarina e Rio Grande do Sul. As florestas plantadas de pinus e eucalipto são a base de suprimento de madeira para estas indústrias. Embora as regióes norte e centro-oeste do país tenham disponíveis extensas áreas de florestas tropicais nativas, poucos estudos tem sido realizados sobre a viabilidade de utilização de madeiras tropicais para produção de painéis aglomerados. $\mathrm{O}$ aproveitamento de resíduos de processamento da madeira provenientes de serrarias e laminadoras na região amazônica pode ser uma alternativa interessante para as indústrias de painéis aglomerados. A disponibilidade deste produto poderia no futuro abrir perspectivas de instalação de pólos moveleiros na região, diminuindo as dependências de importação de móveis de regióes distantes com custos mais elevados.

As espécies de madeira por apresentarem grande variabilidade na sua estrutura anatômica e nas propriedades físicas e químicas, podem exercer influências positivas e negativas na fabricação de produtos colados de madeira. Segundo Marra (1992), as propriedades da madeira possuem forte influência na formação da ligação adesiva e, geralmente, as madeiras de folhosas apresentam mais dificuldades para colagem do que as de coníferas.

A escolha de espécies de madeira para produção de painéis aglomerados deve ser baseada em alguns parâmetros importantes como a densidade, $\mathrm{pH}$ e extrativos (Moslemi 1974; Maloney 1993; Tsoumis 1991). A densidade da madeira é um dos requisitos básicos na escolha de espécies para produção de painéis aglomerados, por influenciar diretamente na sua razão de compactação. Segundo os autores, a razão de compactação, que é a relação entre a densidade do painel e a densidade da madeira, deve ser de no mínimo 1,3 para assegurar uma área de contato satisfatória entre as partículas e densificação suficiente para a formação do painel. Kelly (1977), afirma que, para painéis de mesma densidade, produzidos com madeira de baixa densidade, as suas propriedades mecânicas serão superiores, entretanto, a sua estabilidade dimensional será inferior em comparação aos painéis produzidos com madeira de maior densidade. Segundo o autor, nos painéis com maior razão de compactação, há maior quantidade de partículas de madeira e, consequentemente, ocorrerá maior densificação do painel, resultando em maior inchamento higroscópico da madeira e liberação das tensôes de compressão geradas durante o processo de prensagem à alta temperatura. Maloney (1993) afirma que as espécies com densidade de até $0,55 \mathrm{~g} \cdot \mathrm{cm}^{-3}$ são as mais adequadas para produção de painéis de partículas por atingirem uma razáo de compactação entre 1,3 e 1,6, considerada faixa ideal para o processo de densificação e consolidação do painel até a espessura final. Numa pesquisa conduzida por Vital et al. (1974) sobre painéis aglomerados produzidos com Virola spp., uma espécie de madeira tropical da Amazônia com massa específica aparente (peso e volume seco na estufa) de 0,43 g.cm ${ }^{-3}$, com razão de compactação dos painéis de 1.2:1.0 (baixa) e 1.6:1.0 (alta), os autores encontraram aumentos significativos nos resultados de tração perpendicular e flexão estática (MOE e MOR).

Com relação ao $\mathrm{pH}$ e extrativos presentes na madeira, Marra (1992) afirma que estes parâmetros podem ter influencia direta na cura da resina e, consequentemente, na qualidade dos painéis produzidos. $\mathrm{O}$ pH da madeira pode variar entre 3,0 a 5,5 e de acordo com Kelly (1977), madeiras com $\mathrm{pH}$ muito ácido podem causar a pré-cura da resina uréia-formaldeído durante a fase de fechamento da prensa, prejudicando o grau de adesão entre as partículas e redução nos valores das propriedades mecânicas dos painéis. Madeiras que apresentam $\mathrm{pH}$ pouco ácido requerem quantidade um pouco maior de catalisador para acelerar a cura da resina uréiaformaldeído. A influência dos extrativos na polimerização e cura do adesivo é relatada por vários pesquisadores. Marra (1992) afirma que madeiras com elevados teores de extrativos apresentam dificuldades de colagem resultando em baixa resistência da ligação adesiva entre as partículas.

Tendo em vista a carência de estudos sobre o comportamento das espécies de madeiras da Amazônia na produção de painéis aglomerados, este trabalho teve como objetivo avaliar a qualidade de painéis aglomerados produzidos com nove espécies de madeiras tropicais da Amazônia com base nos resultados de suas propriedades físicas e mecânicas.

\section{MATERIAL E MÉTODOS}

Foram utilizados nesta pesquisa resíduos provenientes de desdobro de toras nas serrarias das seguintes espécies de madeiras tropicais da Amazônia: Scleronema micranthum Ducke (Cardeiro), Ecclinusa guianensis Eyma (Caucho), 
Scleronema sp. (Castanha-de-paca), Copaifera multijuga Hayne (Copaíba), Ocotea sp. (Louro), Ocotea guianensis Aubl (Louroespinho), Caryocar villosum Pers. (Piquiarana), Couratari oblongifolia (Tauari) e Virola surinamensis Rol. Warb (Virola). Os materiais foram coletados na forma de costaneiras nas serrarias da regiáo de Itacoatiara, Estado do Amazonas. Para a manufatura de painéis aglomerados foi utilizada a resina uréia-formaldeído (UF) com teor de sólidos de 65\%, pH 7,8 e viscosidade Brookfield de $450 \mathrm{cP}$. O sulfato de amônia na proporção de $3 \%$ de sólidos em relação aos sólidos de resina foi utilizado como catalisador.

A massa específica aparente da madeira a $12 \%$ de umidade foi determinada através do método estereométrico. A razão de compactação foi determinada através da relação entre a massa específica aparente da madeira e do painel.

Para a manufatura de painéis aglomerados em laboratório, as partículas de madeira foram geradas num picador de disco na forma de flakes, com as dimensóes nominais de $25 \mathrm{~mm}$ (comprimento) x 0,7 mm (espessura) e largura variável. Após a secagem ao teor de umidade médio de $3 \%$, as partículas foram re-processadas no moinho de martelo e classificadas em peneira de malha $0,6 \mathrm{~mm}$ para retirada de finos.

A resina UF líquida catalisada com sulfato de amônia foi aplicada sobre as partículas em quantidade de $8 \%$ de sólidos em relação ao peso seco das partículas. Para a formação do colchão de partículas foi utilizada uma caixa formadora vazada com dimensóes laterais de $50 \mathrm{~cm}$ x $50 \mathrm{~cm}$. Os cálculos para definição de quantidade de materiais para formação de um painel foram feitos para densidade nominal de $0,75 \mathrm{~g} . \mathrm{cm}^{-3}$ e dimensôes de $50 \mathrm{~cm} \times 50 \mathrm{~cm} \times 1,5 \mathrm{~cm}$. Os painéis foram prensados à temperatura de $160^{\circ} \mathrm{C}$, pressão específica de 4,0 MPa e tempo de prensagem de oito minutos. Foram produzidos dois painéis por tratamento, perfazendo um total de dezoito painéis experimentais.

Após a prensagem, os painéis foram esquadrejados e acondicionados em câmara climática com temperatura de 20 $\pm 2{ }^{\circ} \mathrm{C}$ e umidade relativa de $65 \pm 3 \%$, até sua estabilização ao teor de umidade médio de $12 \%$.

Para avaliação das propriedades físico-mecânicas dos painéis foram retirados de cada painel cinco corpos-deprova para ensaios de massa específica aparente, quatro para flexão estática (duas no sentido paralelo e duas no sentido perpendicular ao plano do painel), cinco para traçáo perpendicular (ligação interna) e cinco para absorção de água e inchamento em espessura após duas e 24 horas de imersão em água. As quantidades de corpos-de-prova por espécie (tratamento) foram respectivamente de dez, oito, dez e dez, para cada tipo de ensaios. Os ensaios foram realizados de acordo com os procedimentos descritos na Norma Européia EN 323 (2003), EN 310 (2003), EN 319 (2003) e EN 317 (2003), respectivamente. Os dados obtidos foram submetidos à análise estatística por meio dos testes de Grubb's e Kolmogorov Smirnov, para verificaçáo de outliers e aderência dos dados a distribuição normal. A homocedasticidade foi avaliada por meio do Teste de Cochran e em seguida foram realizadas ANOVA, Análise de Covariância e Teste de médias de Tukey ao nível de probabilidade de 95\%.

\section{RESULTADOS E DISCUSSÃO}

\section{Propriedades físicas dos painéis}

Os resultados de massa específica aparente das espécies, dos painéis e razão de compactação, estão apresentados na Tabela 1.

Os resultados de massa específica aparente das madeiras estudadas variaram na faixa de $0,43 \mathrm{~g} \cdot \mathrm{cm}^{-3} \mathrm{e} 0,68 \mathrm{~g} \cdot \mathrm{cm}^{-3}$, sendo o menor valor obtido para virola e maior valor para piquiarana. Foram constatadas diferenças estatísticas significativas entre as nove espécies estudadas, podendo se definir três grupos distintos de massa específica aparente. Virola, louro-espinho e caucho estão no grupo de baixa massa específica aparente; cardeiro, castanha-de-paca, copaíba, louro e tauri no grupo intermediário; e, a piquiarana que apresentou maior massa específica aparente entre as espécies estudadas.

Com relação à massa específica dos painéis, os valores médios variaram na faixa de $0,67 \mathrm{~g} . \mathrm{cm}^{-3}$ e $0,72 \mathrm{~g} . \mathrm{cm}^{-3}$. Foram constatadas diferenças estatísticas entre os painéis de diferentes espécies, as quais podem ser atribuídas às condições operacionais a nível laboratorial, sem automaçáo e controle de precisão do processo industrial. Os valores obtidos foram inferiores à massa específica aparente nominal calculada de $0,75 \mathrm{~g} . \mathrm{cm}^{-3}$, devido às perdas de materiais durante o manuseio no processo de formação dos painéis e retorno em espessura após prensagem a quente. Tendo em vista as diferenças estatísticas entre as massas específicas aparentes dos painéis, foram realizadas análises de covariância para ajustes de médias das propriedades avaliadas.

Tabela 1 - Valores médios da massa específica aparente das espécies, dos painéis e razão de compactação.

\begin{tabular}{lccc}
\hline Espécie & Mem $\left(\mathrm{g} \cdot \mathrm{cm}^{-3}\right)$ & MEp $\left({\left.\mathrm{g} . \mathrm{cm}^{-3}\right)}^{-}\right.$ & $\mathrm{RC}$ \\
\hline Cardeiro & $0,65 \mathrm{ab}$ & $0,71 \mathrm{abc}$ & $1,09 \mathrm{~d}$ \\
Caucho & $0,47 \mathrm{c}$ & $0,67 \mathrm{bc}$ & $1,44 \mathrm{~b}$ \\
Castanha-de-paca & $0,58 \mathrm{~b}$ & $0,72 \mathrm{a}$ & $1,23 \mathrm{c}$ \\
Copaíba & $0,59 \mathrm{ab}$ & $0,70 \mathrm{abc}$ & $1,19 \mathrm{c}$ \\
Louro & $0,61 \mathrm{ab}$ & $0,67 \mathrm{c}$ & $1,10 \mathrm{~d}$ \\
Louro-espinho & $0,46 \mathrm{c}$ & $0,67 \mathrm{c}$ & $1,46 \mathrm{~b}$ \\
Piquiarana & $0,68 \mathrm{a}$ & $0,69 \mathrm{abc}$ & $1,02 \mathrm{~d}$ \\
Tauari & $0,57 \mathrm{~b}$ & $0,68 \mathrm{ab}$ & $1,20 \mathrm{c}$ \\
Virola & $0,43 \mathrm{c}$ & $0,72 \mathrm{ab}$ & $1,65 \mathrm{a}$ \\
\hline
\end{tabular}

Mem: massa especíica aparente da madeira (12\%); MEp: massa específica dos painéis; RC: razão de compactação. Médias seguidas de mesma letra dentro da coluna são estatisticamente iguais ao nivel de probabilidade de $95 \%$. 
Os valores médios de razão de compactaçáo dos painéis variaram de 1,02 para piquiarana e 1,65 para virola. Apenas três espécies - caucho, louro-espinho e virola apresentaram painéis com razão de compactação acima de 1,3 conforme recomendado por Moslemi (1974) e Maloney (1993). A influência destes resultados de razão de compactaçáo sobre os resultados de propriedades físicas e mecânicas dos painéis será avaliada posteriormente.

Os resultados de absorção de água e inchamento em espessura após duas e 24 horas de imersão em água estão apresentados na Tabela 2.

Os valores médios de absorção de água duas horas e 24 horas variaram respectivamente na faixa de $6,77 \%$ a $17,62 \%$ e $18,43 \%$ a $43,43 \%$. Foram constatadas diferenças estatísticas significativas entre as espécies estudadas. Os painéis produzidos com madeira de caucho, louro, louro-espinho, tauari e virola, apresentaram menor absorçáo de água para duas horas de imersão. Para 24 horas de imersão, as espécies que apresentaram menor absorçấo de água foram: caucho, louro, louro espinho e tauari. A análise dos valores de razáo de compactação dos painéis indica que não houve influência direta desta propriedade sobre os resultados de absorção de água. Painéis de virola e piquiarana com maior e menor razão de compactação respectivamente, não foram os que apresentaram valores extremos de absorção de água. Portanto, a relação direta entre a razão de compactação e absorção de água observada por Vital et al. (1974) para aglomerado de virola não foi confirmada neste estudo.

Os resultados de absorção de água obtidos neste estudo foram bem inferiores aos obtidos por vários pesquisadores. Iwakiri et al. (1996) obtiveram para painéis aglomerados de Pinus taeda e Eucalyptus dunnii, valores médios de absorção de água 24 horas de $75,04 \%$ e $80,05 \%$, respectivamente. Naumann et al. (2008) encontraram para painéis aglomerados

Tabela 2 - Valores médios de absorção de água e inchamento em espessura 2 e 24 horas.

\begin{tabular}{lcccc}
\hline \multirow{2}{*}{ Espécie } & \multicolumn{2}{c}{ AA (\%) } & \multicolumn{2}{c}{ IE (\%) } \\
\cline { 2 - 5 } & duas horas & 24 horas & duas horas & 24 horas \\
\hline Cardeiro & $10,74 \mathrm{~cd}$ & $26,21 \mathrm{~cd}$ & $5,71 \mathrm{ab}$ & $14,70 \mathrm{~d}$ \\
Caucho & $8,16 \mathrm{e}$ & $21,41 \mathrm{de}$ & $5,86 \mathrm{ab}$ & $13,69 \mathrm{de}$ \\
Castanha-de-paca & $15,26 \mathrm{~b}$ & $34,70 \mathrm{~b}$ & $8,45 \mathrm{a}$ & $20,44 \mathrm{ab}$ \\
Copaíba & $17,62 \mathrm{a}$ & $43,43 \mathrm{a}$ & $8,51 \mathrm{a}$ & $18,31 \mathrm{bc}$ \\
Louro & $6,77 \mathrm{e}$ & $18,43 \mathrm{e}$ & $4,01 \mathrm{~b}$ & $10,97 \mathrm{e}$ \\
Louro-espinho & $7,36 \mathrm{e}$ & $22,44 \mathrm{~d}$ & $4,87 \mathrm{~b}$ & $17,57 \mathrm{c}$ \\
Piquiarana & $11,83 \mathrm{c}$ & $28,30 \mathrm{c}$ & $5,56 \mathrm{~b}$ & $15,21 \mathrm{~d}$ \\
Tauari & $8,61 \mathrm{de}$ & $20,85 \mathrm{de}$ & $4,85 \mathrm{~b}$ & $14,02 \mathrm{~d}$ \\
Virola & $9,66 \mathrm{~d}$ & $30,94 \mathrm{c}$ & $8,23 \mathrm{a}$ & $22,37 \mathrm{a}$ \\
\hline
\end{tabular}

AA: absorção de água; IE: inchamento em espessura. Médias seguidas de mesma letra dentro da coluna são estatisticamente iguais ao nível de probabilidade de $95 \%$. de Eucalyptus urophylla e Schizolobium amazonicum, valores médios de $97,2 \%$ e $117,9 \%$, respectivamente. No estudo realizado por Iwakiri et al. (2004) para painéis aglomerados produzidos com Grevilea robusta com densidade de $0,60 \mathrm{~g} \cdot \mathrm{cm}^{-3}$ e $0,80 \mathrm{~g} . \mathrm{cm}^{-3}$, os valores médios de absorção de água 24 horas obtidos foram de $42,91 \%$ e $66,59 \%$, respectivamente.

Os valores médios de inchamento em espessura duas horas e 24 horas variaram respectivamente na faixa de $4,01 \%$ a $8,51 \%$ e $10,97 \%$ a $22,37 \%$. Foram constatadas diferenças estatísticas significativas entre as espécies estudadas. Da mesma forma como em absorção de água, as razôes de compactação dos painéis não afetaram de forma clara os resultados de inchamento em espessura. Os conceitos apresentados por Maloney (1993) e Moslemi (1974) sobre a influência direta da maior razão de compactação sobre o inchamento em espessura, não foram comprovados para os painéis aglomerados produzidos com as nove espécies de madeiras tropicais em estudo. Vital et al. (1974) também não encontraram diferenças no inchamento em espessura 24 horas para painéis aglomerados de virola com razáo de compactação de 1,2 e 1,6 .

Os resultados de inchamento em espessura 24 horas obtidos nesta pesquisa são compatíveis com os valores apresentados na literatura. Iwakiri et al. (1996) encontraram para painéis aglomerados de Pinus taeda e Eucalyptus dunnii, valores médios de 30,50\% e 35,09\%, respectivamente. Naumann et al. (2008) obtiveram para painéis de Eucalyptus urophylla e Schizolobium amazonicum, valores médios de $16,8 \%$ e $17,1 \%$. Os resultados de inchamento em espessura 24 horas, obtidos por Iwakiri et al. (2004), para painéis aglomerados de Grevilea robusta produzidos com densidade de 0,60 e 0,80 g.cm ${ }^{-3}$, foram respectivamente de $17,71 \%$ e $20,88 \%$. Trianoski (2010) encontrou para painéis aglomerados produzidos com madeira de Acrocarpus fraxinifolius, Melia azedarach e Toona ciliata, valores médios de 24,58\%, 17,79\% e 16,78\%, respectivamente.

\section{Propriedades mecânicas dos painéis}

Os resultados de tração perpendicular (TP), módulo de elasticidade (MOE) e módulo de ruptura (MOR) em flexão estática estáo apresentados na Tabela 3.

Os valores médios de tração perpendicular variaram entre 0,68 MPa a 1,46 MPa, para painéis de louro espinho e caucho, respectivamente. Os painéis produzidos com madeira de caucho e tauari apresentaram valores médios de traçáo perpendicular estatisticamente superior em comparação aos painéis de demais espécies. Os valores médios de tração perpendicular obtidos para todas as espécies estudadas atendem ao requisito mínimo da norma européia EN 312:1993 de 0,35 MPa. Os resultados obtidos são indicativos 
Tabela 3 - Valores médios de tração perpendicular, módulo de elasticidade e módulo de ruptura.

\begin{tabular}{lccc}
\hline Espécie & TP $(\mathrm{MPa})$ & $\mathrm{MOE}(\mathrm{MPa})$ & $\mathrm{MOR}(\mathrm{MPa})$ \\
\hline Cardeiro & $1,37 \mathrm{ab}$ & $2.651 \mathrm{abc}$ & $18,70 \mathrm{ab}$ \\
Caucho & $1,46 \mathrm{a}$ & $3.162 \mathrm{a}$ & $27,04 \mathrm{a}$ \\
Castanha-de-paca & $0,92 \mathrm{c}$ & $2.185 \mathrm{c}$ & $13,79 \mathrm{~b}$ \\
Copaíba & $1,27 \mathrm{~b}$ & $2.695 \mathrm{abc}$ & $23,05 \mathrm{a}$ \\
Louro & $1,26 \mathrm{~b}$ & $3.232 \mathrm{a}$ & $23,54 \mathrm{a}$ \\
Louro-espinho & $0,68 \mathrm{~d}$ & $2.677 \mathrm{abc}$ & $12,55 \mathrm{~b}$ \\
Piquiarana & $0,93 \mathrm{c}$ & $2.389 \mathrm{bc}$ & $13,73 \mathrm{~b}$ \\
Tauari & $1,45 \mathrm{a}$ & $2.417 \mathrm{bc}$ & $20,15 \mathrm{a}$ \\
Virola & $0,82 \mathrm{~cd}$ & $2.908 \mathrm{ab}$ & $23,32 \mathrm{a}$ \\
\hline
\end{tabular}

TP: tração perpendicular; MOE: módulo de elasticidade; MOR: módulo de ruptura. Médias seguidas de mesma letra dentro da coluna são estatisticamente iguais ao níve de probabilidade de $95 \%$.

do potencial destas espécies de madeiras tropicais da Amazônia na produçáo de painéis aglomerados.

Com relação aos resultados apresentados na literatura, Trianoski (2010) encontrou para painéis aglomerados produzidos com madeira de Acrocarpus fraxinifolius, Melia azedarach e Toona ciliata, valores de tração perpendicular respectivamente de 1,50 $\mathrm{MPa}, 1,88 \mathrm{MPa}$ e 1,64 $\mathrm{MPa}$. Iwakiri et al. (1996) obtiveram para painéis de Pinus taeda e Eucalyptus dunnii, valores médios de 1,10 MPa e 0,82 MPa, respectivamente. Colli et al. (2010), encontraram para painéis aglomerados produzidos com Schizolobium amazonicum valor médio de tração perpendicular de 0,22 MPa. Já, Vital et al. (1974) encontraram para painéis aglomerados de virola spp com razão de compactação de 1,2:1.0 (baixa) e 1,6:1.0 (alta), valores de traçáo perpendicular de $0,48 \mathrm{MPa}$ e 0,65 $\mathrm{MPa}$, respectivamente. Portanto, os resultados de tração perpendicular obtidos neste estudo estão compatíveis com os valores obtidos para várias espécies de madeiras pesquisadas.

A análise das interaçóes entre a tração perpendicular e razão de compactação indica que não houve uma influência clara desta variável sobre os resultados obtidos. Esta evidência pode ser comprovada nos painéis de virola, que apresentou alta razão de compactação $(1,65)$, entretanto, o seu valor de tração perpendicular de $0,82 \mathrm{MPa}$, foi um dos menores entre as espécies estudadas. Por outro lado, os painéis de cardeiro, com um dos menores valores de razão de compactação de 1,09, apresentou tração perpendicular de 1,37 MPa.

Os valores médios de módulo de elasticidade (MOE) variaram na faixa de $2.185 \mathrm{MPa}$ para painéis de castanha-dopará e 3.232 MPa para painéis de louro. Os painéis produzidos com madeira de caucho e louro apresentaram valores médios de MOE estatisticamente superior em comparação aos painéis de castanha-de-paca, piquiarana e tauari e iguais em relação aos painéis de demais espécies. Os valores médios de MOE de todas as espécies estudadas atendem ao requisito mínimo da norma européia EN 312:1993 de 1.600 MPa. A análise das interaçôes entre o MOE e razão de compactação demonstram que náo houve influência direta desta variável sobre os resultados obtidos. Esta relação pode ser evidenciada nos painéis de louro e caucho, os quais apresentaram maiores valores de $\mathrm{MOE}$, entretanto, os valores de razão de compactação de 1,10 e 1,44 foram bastante diferenciados. Vital et al. (1974) encontraram para painéis de virola com razão de compactação de 1,2 e 1,6, aumento significativo de $\mathrm{MOE}$, cujos valores foram respectivamente de $2.233 \mathrm{MPa}$ e $3.598 \mathrm{MPa}$.

Os resultados obtidos neste estudo foram superiores aos apresentados por Naumann et al. (2008) para painéis de Eucalyptus urophylla e Schizolobium amazonicum, com valores médios respectivamente de $734 \mathrm{MPa}$ e $1.873 \mathrm{MPa}$. Iwakiri et al. (1996) obtiveram para painéis de Pinus taeda e Eucalyptus dunnii, valores médios de MOE de 2.269 MPa e 2.312 MPa, respectivamente. Trianoski (2010) encontrou para painéis de Acrocarpus fraxinifolius, Melia azedarach e Toona ciliata, MOE médio de 2.134 MPa, 2.191 MPa e 2.427 MPa, respectivamente.

Os valores médios de módulo de ruptura (MOR) variaram na faixa de 12,55 MPa para painéis de louro-espinho e 27,04 $\mathrm{MPa}$ para painéis de caucho. Os painéis produzidos com madeira de castanha-de-paca, louro espinho e piquiarana apresentaram valores médios de MOR estatisticamente inferior em comparação aos painéis de demais espécies. Com exceção de louro espinho, os valores médios de MOR de todas as demais espécies estudadas atendem ao requisito mínimo da norma européia EN 312:1993 de $13 \mathrm{MPa}$. Assim como foi verificado para as outras propriedades, não foram constatadas influências claras da razão de compactação sobre os resultados de MOR, pois os painéis de louro espinho que apresentaram alta razão de compactação (1.46), obteve menor valor de MOR.

Os resultados de MOR obtidos nesta pesquisa estão compatíveis com os valores referenciais apresentados na literatura. Iwakiri et. al. (1996) encontraram para painéis aglomerados de Pinus taeda e Eucalyptus dunnii, valores médios de MOR de 16,94 MPa e 15,86 MPa, respectivamente. Cabral et al. (2007) obtiveram para painéis aglomerados produzidos com maravalhas de Eucalyptus grandis, mistura de Eucalyptus urophylla e Pinus sp, mistura de Eucalyptus cloeziana e Pinus $s p$, valores médios de MOR de 15,52 MPa, 16,77 MPa e 17,92 MPa, respectivamente. Trianoski (2010) obteve para painéis de Acrocarpus fraxinifolius, Melia azedarach e Toona ciliata, MOR médio de 18,19 MPa, 18,56 MPa e 19,83 $\mathrm{MPa}$, respectivamente. Já, Vital et al. (1974) encontraram para painéis de virola com razão de compactação de 1,2 e 1,6, valores médios de MOR de 14,68 $\mathrm{MPa}$ e 25,36 $\mathrm{MPa}$, respectivamente. 


\section{CONCLUSÕES}

Com base nos resultados obtidos nesta pesquisa as seguintes conclusóes podem ser apresentadas:

- Os resultados das propriedades físicas e mecânicas dos painéis aglomerados produzidos com as nove espécies de madeiras tropicais foram satisfatórios em comparação aos valores referenciais apresentados na literatura para espécies de madeiras provenientes de floretas plantadas;

- As razóes de compactação dos painéis aglomerados não influenciaram de forma clara nas suas propriedades físicas e mecânicas;

- Os valores médios de tração perpendicular, módulo de elasticidade e módulo de ruptura obtidos para todas as espécies estudadas atendem aos requisitos mínimos da norma européia EN 312:1993;

- As avaliaçóes dos resultados de ensaios obtidos nesta pesquisa indicam o potencial de utilizaçáo das nove espécies provenientes de florestas tropicais da Amazônia na produçáo de painéis de madeira aglomerada, com destaque para Ecclinusa guianensis Eyma (Caucho) que, de uma forma geral, apresentou melhores resultados de propriedades físicomecânicas.

\section{AGRADECIMENTOS}

Os autores expressam seus agradecimentos à pesquisadora do INPA, Dra. Claudete Catanhede do Nascimento, pela concessão do material de pesquisa viabilizada através do projeto "Caracterização dos resíduos madeireiros e desenvolvimento de tecnologia para seu aproveitamento", financiado pela FINEP. Agradecimentos também ao apoio recebido no âmbito do projeto "Estudos tecnológicos de alternativas de uso de resíduos florestais na Amazônia central", financiado pelo Edital CT-Amazônia do CNPq.

\section{BIBLIOGRAFIA CITADA}

BNDES. 2008. Tecnhical Report. Wood panels in the Brazil: overview and perspectives. Rio de Janeiro. p. 121-156 (In Portuguese).

Cabral, C.P.; Vital, B.R.; Lucia, R.M.D.; Pimenta, A.S. 2007. Properties of particleboard manufactured with mixed particles from Eucalyptus spp and Pinus elliottii. Revista Árvore, 31: 897905 (in Portuguese, with abstract in English).

Colli, A.; Vital, B.R.; Carneiro, A.C.O.; Silva, J.C.; Carvalho, A.N.M.L.; Della Lucia, R.M. 2010. Properties of panels manufactured with wood particles of paricá (Schizolonium amazonicum Huber ex.Ducke) and coconut fibers. Revista Árvore, 34: 333-338 (in Portuguese, with abstract in English).

EUROPEAN COMMITTEE FOR STANDARDIZATION. Norma EN 310. Determination of modulus of elasticity in bending and bending strength. Bruxelas. 2003.
EUROPEAN COMMITTEE FOR STANDARDIZATION. Norma EN 312. Particleboard - Specifications. Bruxelas. 1993.

EUROPEAN COMMITTEE FOR STANDARDIZATION. Norma EN 317. Determination of swelling in thickness after immersion in water. Bruxelas. 2003.

EUROPEAN COMMITTEE FOR STANDARDIZATION. Norma EN 319. Determination of internal bond. Bruxelas. 2003.

EUROPEAN COMMITTEE FOR STANDARDIZATION. Norma EN 323. Determination of board density. Bruxelas. 2003.

Iwakiri, S.; Latorraca, J.V.F.; Silva, D.A.; Gabardo, J.L.; Klitzke, R.J.; Fofano, A.; Fabrowski, F.; Interanmense, M.T. 1996. Particleboard manufacture from Pinus elliottii (Engelm) and Eucalyptus dunnii (Maid). Agrárias, 15: 33-41 (in Portuguese, with abstract in English).

Iwakiri S.; Shimizu, J.; Silva, J.C.; Del Menezzi, C.H.S; Puehringer, C.A.; Venson, I. Laroca, C. 2004. Particleboard manufacture from Grevilea robusta A. Cunn. Ex R. Br. Revista Árvore, 28 : 56-60 (in Portuguese, with abstract in English).

Kelly, M.W. 1977. Critical literature review of relationships between processing parameters and physical properties of particleboard. USDA Forest Service General Technology Report Forest Products Laboratory, Madison, USA. 66pp.

Maloney, T.M. 1993. Modern particleboard and dry-process fiberboard manufacturing. M. Freeman, San Francisco, USA. 689pp.

Moslemi, A.A. 1974. Particleboard vol. 1: Materials. Southern Illinois University Press, London. 244pp.

Marra, F.S. 1992. Tecnhonology of wood bonding - Principles in practice. Van Nostrand Reinhold, New York. 453pp.

Naumann. R.B.; Vital, B.R.; Carneiro, A.C.O.; Della Lucia, R.M.; Silva, J.C.; Carvalho, A.M.M.L.; Colli, A. 2008. Properties of particleboard manufactured from Eucalyptus urophylla S.T.Blake and Schizolobium amazonicum Herb. Revista Arvore, 32: 11431150 (in Portuguese, with abstract in English).

Trianoski, R. 2010. Avaliaçâo do potencial de espécies florestais alternativas de rápido crescimento para produção de painéis de madeira aglomerada. 2010. Dissertaçáo de mestrado, Universidade Federal do Paraná, Curitiba, Paraná. 260pp (in Portuguese, with abstract in English).

Tsoumis, G. 1991. Science and technology of wood: structure, properties and utilization. Van Nostrand Reinhold, New York, USA. 494pp.

Vital, B.R.; Lehmann, W.F.; Boone, R.S. 1974. How species and board densities affect properties of exotic hardwood particleboards. Forest Products Journal, 24: 37-45.

Recebido em 06/10/2010

Aceito em 04/04/2011 